\title{
Eletroterapia IVL no tratamento da covid-19 e sequelas no sistema nervoso central
}

\author{
Daniela Oliveira Lopes ${ }^{1}$ \\ daniela.lopexx@hotmail.com
}

\author{
Dr. Fabiano de Abreu Agrela Rodrigues ${ }^{2}$ \\ deabreu.fabiano@gmail.com
}

\begin{abstract}
RESUMO
Pacientes internados por causa da Covid-19 foram tratados com eletroterapia e obtiveram bons resultados em relação subsequente recuperação. Dois hospitais do Brasil utilizaram as correntes elétricas na recuperação de pacientes que ficaram entubados por longos períodos na UTI. O aparelho emissor de terapia infravermelho longo ativa trilhões de células por minuto, eliminando ácido lático e toxinas e promovendo uma melhoria na circulação de oxigênio e o aumento do sistema imunológico por oxigenação celular.
\end{abstract}

Palavras-chaves: covid-19, sars-cov, eletroterapia, tratamento, ivl, infravermelho, infravermelho longo

\footnotetext{
${ }^{1}$ Especialista em estética e cosmetologia intradermicas Representante da Sociedade Brasileira de Estética e Cosmetologia Escola Superior de Estética e Cosmetologia ESEC

Rua Tabapua, 145 - 11 andar, Bairro Itaim Bibi São Paulo SP CEP: 043533-010 BRASIL

${ }^{2} \mathrm{PhD}$, neurocientista, neuropsicólogo, mestre em psicanálise com pós graduação em antropologia, jornalista e especialista em nutrição clínica.

CPAH- Centro de Pesquisas e Análises Heráclito Rua Costinha, n95 4550-023 Sobrado e Bairros Aveiro, Castelo de Paiva PORTUGAL
} 


\title{
IVL electrotherapy in the treatment of Covid-19 and central nervous system sequelaes
}

\begin{abstract}
Patients admitted for Covid-19 were treated with electrotherapy and obtained good results in relation to recovery. Two hospitals in Brazil used electrical currents to recover patients who had spent many days in the ICU. The long infrared therapy emitting device activates trillions of cells per minute, eliminating lactic acid and toxins, promoting an improvement in oxygen circulation and an increase in the immune system through cellular oxygenation.
\end{abstract}

Keywords: covid-19, sars-cov, electrotherapy, treatment

Artículo recibido: 02 enero 2022 Aceptado para publicación: 28 enero 2022 Correspondencia: deabreu.fabiano@gmail.com Conflictos de Interés: Ninguna que declarar 


\section{INTRODUÇÃO}

A eletroterapia consiste no uso de correntes elétricas dentro da terapêutica estética. Os aparelhos de eletroterapia utilizam uma intensidade de corrente muito baixa: miliamperes e microamperes. Os eletrodos são aplicados diretamente sobre a pele e o organismo funciona como condutor. Na eletroterapia devem-se considerar parâmetros como: resistência, intensidade, voltagem potência e condutividade.

\section{A frequência das correntes utilizadas na eletroteparia são classificadas em:}

1. Baixa Frequência: 1 a $1.000 \mathrm{~Hz}$, mais utilizada na prática clínica a faixa de 1 a 200 $\mathrm{Hz}$;

2. Corrente Galvânica, Farádica, Diadinâmicas, TENS (Estimulação Nervosa Elétrica Transcutânea) e FES (Estimulação Elétrica Funcional);

3. Média Frequência: 1.000 a $10.000 \mathrm{~Hz}$, sendo utilizado na eletroterapia de $2.000 \mathrm{a}$ 4.000 Hz. Interferencial e Corrente Russa;

4. Alta Frequência: $10.000 \mathrm{~Hz}$ a $100.000 \mathrm{~Hz}$. Ondas Curtas, Micro-ondas, Ultrassom (Ultrassom).

A utilização de correntes produz movimento e altera a temperatura do organismo, trazendo efeitos fisiológicos e bioquímicos tais como: aumento da oxigenação e hidratação dos tecidos (fundamental em tratamentos para rejuvenescimento), elevação da velocidade de cicatrização (como no caso de cicatrizes), estímulo do colágeno, melhora da circulação local, entre outros.

A eletroterapia também pode ocorrer por sonidoterapia (terapia por meio de ondas). $\mathrm{O}$ método alivia, de maneira eficaz, a dor nas articulações profundas e nos tecidos musculares. Seu calor intenso amacia e alonga o tecido conjuntivo, aumentando a mobilidade e o pleno uso das articulações. O número de ondas que determinam a frequência do aparelho são $3 \mathrm{MHz}$ (também podem ser utilizados na estética) e $1 \mathrm{MHz}$. Cabe destacar que as ondas podem penetrar com mais facilidade em alguns tecidos, conforme sua constituição e densidade.

Assim, regiões com muitos pelos e queratina como a planta dos pés, dificultam a absorção. O tipo de cabeçote também pode influenciar a absorção do tecido, por exemplo, cabeçotes de $1 \mathrm{MHz}$ são absorvidos de até $5 \mathrm{~cm}$ de profundidade, enquanto os de $3 \mathrm{MHz}$ são absorvidos de 1,5 a $3 \mathrm{~cm}$. 
As ondas terapêuticas se dividem em dois tipos: Contínuo - não possui interrupções no fluxo longitudinal das ondas e, normalmente, são indicadas para as lesões crônicas; e Pulsátil - que possui interrupções no fluxo contínuo de ondas ultrassônicas; as vibrações são interrompidas por pausas e são indicadas para lesões agudas.

As vibrações mecânicas da eletroterapia produzem um aumento do metabolismo local, gerando aumento do fluxo sanguíneo melhorando a nutrição tecidual, a retirada de catabólitos e favorecendo a regeneração tecidual. $\mathrm{O}$ aumento do metabolismo local e a consequente retirada dos catabólitos levam a uma descompressão das terminações nervosas. A ação mecânica entre os tecidos produz a liberação de aderências devido à separação das fibras de colágenos, remodelagem das camadas intracelular, absorção de excesso de íons de $\mathrm{Ca}++$.

Sobre a estimulação galvânica, pesquisas mostram que esta é mais eficaz durante os estágios iniciais de tratamento quando combinada com outras formas de terapia como gelo, calor e exercícios de reforço e de amplitude de movimento, é um aparelho de aplicação de corrente elétrica direta. A corrente galvânica, chamada de voltaica ou contínua, é unidirecional e essencialmente polarizada, mantendo seus polos bem determinados durante todo o tratamento. Na área estética, esta corrente é utilizada para introduzir na pele determinadas substâncias consideradas curativas.

A corrente galvânica possui a propriedade de determinar efeito térmico, químico e efeitos fisiológicos e de formar um campo magnético. Ao atravessar os tecidos do corpo humano produz uma cadeia de fenômenos físico-químicos cujos fundamentos se manifestam especialmente na dissociação molecular. Como, por exemplo, o cloreto de sódio existente em nosso organismo ao se dissociar forma íons $\mathrm{Na}$ e $\mathrm{Ci}$. A iontoforese (ionização) se resume no aproveitamento desses fenômenos físico- químicos com a finalidade de fazer penetrar substâncias terapêuticas através de pele íntegra. O ionizador galvânico constante com produto químico colocado no eléctrodo negativo é colocado em cima do depósito de gordura e o elétrodo positivo paralelo, produz uma contratura local, fazendo penetrar o produto químico com suas partículas elétricas no interior do depósito de gordura (tratamento localizado).

Com a corrente galvânica são realizadas as seguintes técnicas faciais: ionização, desincruste, eletrolifting ou galvanopuntura, eletrólise ou eletrocoagulação, cujos efeitos fisiológicos são: hiperemia local (produção de calor), vasodilatação local, elevação da 
temperatura local, elevação do metabolismo, otimização da circulação sanguínea, analgesia.

A eletroterapia realizada com aparelho de infravermelhor longo ativa trilhões de células por minuto, eliminando ácido lático, toxinas através da sudorese, melhora da circulação de retorno comprometida, aumento dosistema imune por oxigenação celular. O aparelho atua em três comprimentos de onda: curta, média e infravermelho longo, medidos em mícrons ou micrômetros. Os raios infravermelhos longos oferecem a maioria dos benefícios de saúde, porque são capazes de penetrar de cinco a sete centímetros no corpo, aumentando suavemente a temperatura da superfície do corpo. A energia do raio infravermelho longo, ativada pelo calor, é absorvida pelas células humanas em um processo conhecido como "ressonância". A temperatura celular varia de $42^{\circ}, 46^{\circ}, 50^{\circ}$ a $60^{\circ}$.

\section{TRATAMENTO EM PACIENTES DE COVID-19}

Após diagnóstico de Sepse (infecção no sangue) e ser tratada por meio de eletroterapia, no qual obteve êxito, não perdi tempo e iniciei pesquisas sobre a utilização da câmara de infravermelho longo IVL na recuperação de pacientes que tiveram Covid-19.

Durante os estudos, identifiquei que o aparelho emissor de terapia infravermelho longo ativa trilhões de células por minuto, eliminando ácido lático e toxinas, promovendo a melhora da circulação de oxigênio e aumento do sistema imunológico por oxigenação celular. Minhas descobertas já foram testadas em 18 pacientes e os resultados obtidos foram surpreendentes.

Os pacientes foram submetidos a sessões de infravermelho longo e corrente galvânica durante quatro semanas, com tratamento três vezes por semana, duração de 50 minutos cada aplicação. O protocolo utilizado com infravermelho longo fotonplatina dinâmico, da seguinte forma:

- 50 minutos aplicação de infravermelho em todo o corpo, ficando apenas a cabeça sem receber emissão de infravermelho.

- após a terapia com infravermelho, 15 minutos de ionização, circulando em todo o corpo a corrente galvânica.

- ao final dos primeiros 60 minutos, da aplicação, todos os pacientes sentiram melhora significativa de $50 \%$ do estado de debilitação no qual se encontrava.

- em casa todos os pacientes utilizaram oleo essencial, que é o estudo de caso do 
proximo artigo.

Na segunda aplicação os pacientes obtiveram o mesmo protocolo da primeira aplicação. Após 7 dias da primeira aplicação do tratamento todos os pacientes se encontravam sem nenhum sintoma do Covid-19. As demais aplicações seguiram o rito normalmente, a fim de aumentar o sistema imunológico.

Ao final da primeira aplicação, todos os pacientes sentiram melhora significativa de $50 \%$ do estado de debilitação no qual se encontravam, e após 7 dias da primeira aplicação, todos os pacientes estavam sem nenhum sintoma da Covid-19.

O método de tratamento consiste no uso de correntes elétricas aplicadas diretamente sobre a pele e o organismo será o condutor. Os aparelhos de eletroterapia utilizam uma intensidade de corrente muito baixa. Os raios infravermelhos longos oferecem a maioria dos benefícios de saúde, porque são capazes de penetrar de cinco a sete centímetros no corpo, aumentando suavemente a temperatura da superfície do corpo. A energia do raio infravermelho longo, ativada pelo calor, é absorvida pelas células humanas em um processo conhecido como "ressonância".

\section{HOSPITAIS UTILIZAM A ELETROTERAPIA EM PACIENTES DE UTI}

O Hospital Regional de Chapadinha, da rede estadual de saúde do Maranhão, também utilizou a estimulação por eletroterapia para recuperação muscular em pacientes internados por complicações da Covid-19. O recurso gerou bons resultados, em especial em pacientes que passaram por longos períodos de sedação e intubação, cuja musculatura perdeu o tônus em função do não uso e dos bloqueadores neuromusculares.

Os pacientes de longa internação costumam ficar incapacitados de realizar movimentos simples. Por isso, além da fisioterapia respiratória, outro recurso no tratamento tem sido a eletroterapia, que consiste em aplicar correntes elétricas diversas para conseguir efeitos como analgesia, diminuição de edema, relaxamento e fortalecimento muscular, para auxiliar no processo de reabilitação. O tratamento utilizou a corrente FES (Estimulação Elétrica Funcional), uma corrente alternada de baixa frequência, que provoca contrações musculares através de eletrodos sobre a pele do paciente.

O Hospital Vera Cruz, de Belo Horizonte (MG) lançou mão do sistema ReCARE na recuperação precoce de pacientes em UTI. A estimulação elétrica neuromuscular é uma importante ferramenta para promover ganho de força, tratar a disfunção de vários nervos do paciente e a fraqueza adquirida na unidade de terapia intensiva (UTI). Com estes 
objetivos, o ReCARE - Sistema de Ativação e Monitorização Neuromuscular (SAMNA) tem revolucionado a recuperação de pacientes, tanto internados por Covid-19 quanto por outras doenças, contribuindo para a redução do tempo de internação na UTI. A partir da ativação e monitorização automática de vários grupos musculares, o ReCARE torna a técnica de eletroestimulação eficaz e viável potencializandoos resultados dos médicos, fisioterapeutas e equipe de enfermagem na UTI. O hospital utiliza intensamente a tecnologia ReCARE para terapias com estimulação elétrica neuromuscular em pacientes acamados em leitos da UTI com Covid-19 e lesões neurais. Além de oferecer ganho de força muscular, o sistema pode ser uma ferramenta para reduzir o tempo de ventilação mecânica desses pacientes.

\section{SEQUELAS DA COVID-19 NO SISTEMA NERVOSO CENTRAL}

Segundo o neurocientista Fabiano de Abreu, a Covid-19 pode deixar sequelas no sistema nervoso central dos pacientes, sendo que a maior preocupação recai tanto nos danos físicos da doença como nos traumas que esta acarreta em âmbito psicológico. A doença pode provocar danos neurológicos em nível celular ou a própria infecção pode causar traumas que afetam a capacidade cognitiva e que podem resultar em transtornos, síndromes ou outras variáveis futuras. Os danos que o coronavírus pode causar estão ainda em fase incipiente de estudo, uma vez que é uma doença relativamente nova, mas, a cada dia, acrescem os casos de relatos de pessoas que, mesmo após superarem, referem sequelas. Pacientes com Covid-19, mesmo recuperados, ainda sofrem com a mudança no paladar e olfato, que pode ser irreversível. Isso está relacionado à lesão causada, principalmente, nos neurônios sensoriais, primários.

A Covid-19 tornou-se rapidamente uma das pandemias mais proliferantes e mortais da história moderna. A doença é uma infeção aguda e tem seus efeitos predominantemente localizados nos pulmões mas também em todo o sistema cardiovascular e o sistema nervoso. Os sintomas são vários como, por exemplo, dores de cabeça, musculares e articulares, fadiga e "cérebro neblina", além de perda do paladar e olfato, que podem durar semanas ou mesmo meses. Em casos mais graves, a Covid-19 causa encefalite ou acidente vascular cerebral. É, portanto, irrefutável que afeta o sistema nervoso central e tem efeitos neurológicos inegáveis.

Estudos em laboratórios apontam que o novo coronavírus tem a capacidade de penetrar nas células nervosas. Os sintomas neurológicos mais persistentes em pacientes infetados 
tendem a ser menos graves, mas não tão fáceis de descobrir e completar um quadro clínico único e, muitos pacientes experimentam perda de memória, desorientação e confusão mental. Os estudos indicaram que pacientes apresentaram acidente vascular cerebral isquémico, hemorragia, seio venoso trombose, vasculite cerebral, encefalopatia, encefalite, convulsões, síndromes neuropsiquiátricas, síndrome de Guillain Barré, Miller Síndrome de Fisher, neuromiopatia etc.

Outro sintoma que ocorre com frequência em pacientes infetados é a anosmia ou perda do olfato. A perda da capacidade de sentir e distinguir cheiros pode ter origem em mudanças que acontecem sem que os próprios nervos se tornem infetados. A comunidade científica ainda investiga como a perda do olfato pode resultar de uma interação entre o vírus e outro recetor nos neurônios olfatórios ou de seu contato com células não neurais que revestem o nariz. Desta maneira, podemos concluir que o vírus não precisa se espalhar dentro dos neurônios para causar alguns dos sintomas neurológicos misteriosos que emergem com a doença.

Theodore Price, um cientista da Universidade de Dallas (Texas), salientou que embora os sintomas comuns iniciais como dor de garganta, dores de cabeça, dores musculares em todo o corpo e tosse severa são mais prevalentes, existem outros sintomas curiosos que permitem algumas considerações. Para determinar se a Covid- 19 pode infetar células de todo o corpo, o estudioso e sua equipe determinaram que o principal critério é a presença da enzima conversora angiotensina 2 (ACE2), uma proteína incorporada na superfície das células.

ACE2 atua como um recetor que envia sinais à célula para regular a pressão arterial, sendo um ponto de entrada do novo coronavírus.

Price e sua equipe obtiveram células nervosas doadas após a morte ou cirurgias de câncer, e mais tarde o os pesquisadores realizaram o sequenciamento do RN. Esta é uma técnica para determinar quais proteínas uma célula está prestes a produzir usando anticorpos para direcionar o próprio ACE2. Eles descobriram que um subconjunto de DRG neurônios continham ACE2, fornecendo ao vírus um portal para as células. Os neurônios sensoriais têm longos links chamados axônios, cujas terminações parecem estímulos específicos no corpo e, em seguida, transmiti-los ao cérebro na forma de sinais eletroquímicos. Os neurônios DRG específicos que continha ACE2 também tinha as instruções genéticas, RNA, para uma proteína sensorial chamada MRGPRD. Esta proteína marca as células 
como um subconjunto de neurônios cujas terminações estão concentradas nas superfícies do corpo - a pele e os órgãos internos, incluindo os pulmões - onde eles seriam posicionados para capturar o vírus. Desta forma, a infeção do nervo pode contribuir para doenças agudas e também sintomas duradouros.

Recentemente, estão sendo desenvolvidos estudos que pretendem relacionar os sistema nervoso com o sistema cardiovascular. É a ampliação de uma visão integrada da neurociência com o sistema cardiovascular e como as patologias mentais podem afetar outros sistemas vitais, como o sistema cardiovascular em situações como a atual pandemia. Embora ainda não existam evidências científicas irrefutáveis de que a entrada direta do vírus nas células nervosas é o principal mecanismo que causa danos no nível celular, a comunidade científica não deve desconsiderar esta possibilidade. Por outro lado, existe uma grande possibilidade de que condições inflamatórias externas às células nervosas podem alterar sua atividade. Na pior das hipóteses, eles podem até causar permanentes danos.

Em outra perspetiva de abordagem do problema está a possibilidade de que a interação entre as partículas virais e os próprios neurônios pode levar a um ataque autoimune aos nervos. Levando em consideração de que todo e qualquer patógeno que interfira com o neurônios causam danos irreversíveis, mesmo que os neurônios não sejam assim significativos, chegamos à conclusão de que o Covid-19, ao afetar os neurônios independentes causam traumas irreversíveis que afetam a cognição e podem resultar em um distúrbio ou síndrome. Por fim, conhecer os tipos de consequências será possível no futuro avaliando pessoas que sofreram a doença.

\section{CONCLUSÃO}

Tratamentos de eletroperativa com raios infravermelhos surtiram o efeito desejado em pacientes de Covid-19 que ficaram muito tempo entubados nas UTIs. Experiências demonstraram que o recurso gerou bons resultados na recuperação da musculatura que perdeu o tônus em função do não uso e dos bloqueadores neuromusculares. O método de tratamento consiste no uso de correntes elétricas aplicadas diretamente sobre a pele e o organismo será o condutor. Os aparelhos de eletroterapia utilizam uma intensidade de corrente muito baixa. Os raios infravermelhos longos oferecem a maioria dos benefícios de saúde, porque são capazes de penetrar de cinco a sete centímetros no corpo, aumentando suavemente a temperatura da superfície do corpo. A energia do raio infravermelho longo, 
ativada pelo calor, é absorvida pelas células humanas em um processo conhecido como "ressonância".

Em relação às sequelas que a Covid-19 provoca nos doentes, cabe ressaltar que a doença pode provocar danos neurológicos em nível celular ou a própria infeção pode causar traumas que afetam a nossa capacidade cognitiva e que podem resultar em transtornos, síndromes ou outras variáveis futuras. A doença é uma infeção aguda e tem seus efeitos predominantemente localizada nos pulmões, mas também em todo o sistema cardiovascular e o sistema nervoso. Os sintomas são vários como, por exemplo, dores de cabeça e musculares e articulares, fadiga e "cérebro neblina", além de perda do paladar e olfato, que podem durar semanas ou mesmo meses. Em casos mais graves, a Covid-19 causa encefalite ou acidente vascular cerebral. É, portanto, irrefutável que afeta o sistema nervoso central e tem efeitos neurológicos inegáveis.

\section{REFERÊNCIAS}

PRICE et al. 2020. Uma interação farmacológica entre COVID-19 amostras de pacientes e neurônios sensoriais humanos revelam potencial condutores de disfunção pulmonar neurogênica, cérebro, comportamento, and Immunity, Volume 89, outubro, páginas 559- 568

Eletroterapia é utilizada em Hospital de Chapadinha em recuperação de pacientes com Covid-19. Disponível em 3/3/2021 e acessado em 15/03/2021. Eletroterapia é utilizada em Hospital de Chapadinha em recuperação de pacientes com Covid-19 - Maranhão de Todos Nós (www.ma.gov.br)

Eletroterapia neuromuscular na recuperação de pacientes da COVID-19. Disponível em 20/10/2020 e acessado em 17/03/2021. Eletroterapia neuromuscular na recuperação de pacientes da COVID-19 - Visuri

O que é Eletroterapia e para que serve? Disponível em 20/03/2019 e acessado em 17/03/2020. O que é Eletroterapia e para que serve? (hsmed.com.br) 